\title{
Risk Adjustment Using Administrative Data
}

\author{
Impact of a Diagnosis-type Indicator \\ William A. Ghali, MD, MPH, Hude Quan, MD, PhD, Rollin Brant, PhD
}

\begin{abstract}
OBJECTIVES: To determine the frequency with which commonly coded clinical variables are complications, as opposed to baseline comorbidities, and to compare the results of 2 risk-adjusted outcome analyses for coronary artery bypass graft surgery for which we either (a) ignored, or (b) used the available "diagnosis-type indicator."
\end{abstract}

DESIGN: Analysis of existing administrative data.

SETTING: Twenty-three Canadian hospitals.

PATIENTS: A total of 50,357 coronary artery bypass graft surgery cases.

MEASUREMENTS AND MAIN RESULTS: Among 21 clinical variables whose definitions involve the diagnosis-type indicator, 14 were predominantly ( $\geq 97 \%$ ) baseline risk factors when present. Seven variables were often complication diagnoses: renal disease (when present, $13 \%$ coded as complications), recent myocardial infarction (15\%), peptic ulcer disease $(15 \%)$, congestive heart failure $(17 \%)$, cerebrovascular disease $(26 \%)$, hemiplegia $(34 \%)$, and severe liver disease (35\%). The results of risk adjustment analyses predicting in-hospital mortality differed when the diagnosistype indicator was either used or ignored, and as a result, adjusted hospital mortality rates and rankings changed, often dramatically, with rankings increasing for 10 hospitals, decreasing for 9 hospitals, and remaining the same for only 4 hospitals.

CONCLUSIONS: The results of analyses performed using the diagnosis-type indicator in Canadian administrative data differ considerably from analyses that ignore the indicator. The widespread introduction of such an indicator should be considered in other countries, because risk-adjustment analyses performed without a diagnosis-type indicator may yield misleading results.

KEY WORDS: administrative data; risk adjustment; complications; comorbidities; coronary artery bypass graft surgery.

J GEN INTERN MED 2001;16:519-524.

A dministrative hospital discharge data have been, and will continue to be, used to measure outcomes and quality of care because of their generally wide availability,

Received from the Department of Medicine (WAG) and the Department of Community Health Sciences (WAG, HQ, RB), Faculty of Medicine, University of Calgary, Calgary, Alberta, Canada.

Presented at the $22^{\text {nd }}$ Annual Meeting of the Society of General Internal Medicine, San Francisco, Calif, April 29-May 1, 1999.

Address correspondence and reprint requests to Dr. Ghali: 3330 Hospital Dr. N.W., Calgary, Alberta, Canada T2N 4N1 (e-mail: wghali@ucalgary.ca). relatively low cost, and broad geographic coverage. ${ }^{1}$ Weighing heavily against the positive attributes of administrative data, however, are a number of concerns regarding the validity and quality of administrative data sources. One frequently cited limitation of administrative data is the lack of information on the sequencing of clinical diagnoses. ${ }^{1}$ This is problematic in risk-adjusted outcome analyses across providers, because it is difficult to distinguish comorbidities present at baseline, factors that should be accounted for in outcome analyses to protect providers who care for sicker patients, from complications arising later on.

Unlike many administrative hospital discharge databases, Canadian hospital discharge data contain a single digit "diagnosis-type indicator" that accompanies each coded diagnosis. ${ }^{2}$ This indicator distinguishes between diagnoses present at the time of hospital admission and diagnoses arising after hospitalization. The diagnosis-type designations include: type $\mathrm{M}$, most responsible diagnosis; type 1, primary comorbid conditions present at admission (and playing a role during the hospitalization); type 2, complication diagnoses arising some time after hospital admission; type 3 , secondary comorbid conditions present at admission but not playing an important role during hospitalization; type 4, morphology codes; and type 9, E-code diagnoses from the International Classification of Diseases, 9th revision (ICD-9). ${ }^{3}$

In recent work evaluating Canada-wide risk-adjusted outcomes of coronary artery bypass graft (CABG) surgery, 4,5 we used this diagnosis-type indicator field to exclude complication diagnoses from measurements of baseline severity of illness. Here, we present data on the frequency with which various coded clinical variables were actually complications, as opposed to baseline comorbidities, in our data. We also compare the results of two risk-adjusted CABG surgery hospital outcome analyses for which we either (a) ignored or (b) used the available diagnosis-type indicator.

\section{METHODS}

Data for this study were obtained from the Canadian Institute for Health Information (CIHI), an agency that compiles hospital discharge data from all Canadian provinces except Quebec. Cases were identified by screening all hospital discharges across Canada from fiscal years $1992 / 1993$ through 1995/1996 for CABG surgery procedure codes 48.11 through 48.19 , defined according to the Canadian Classification of Procedures (CCP). ${ }^{6}$ The Canadian hospital discharge data contain basic demographic information (age, gender, and postal code), urgency of admission, information on in-hospital outcomes (length of 
stay, special care unit use, and mortality), a total of 16 diagnosis codes defined according to the ICD-9, ${ }^{3}$ and 10 procedure codes defined according to the CCP.

The coded diagnoses in each discharge record were used to define 21 clinical variables that are potentially influenced by the diagnosis-type indicator. The widely cited Deyo adaptation of the Charlson comorbidity index ${ }^{7,8}$ was used to define 17 of the 21 variables (e.g., congestive heart failure, renal disease). These 17 variables defined by the Deyo coding scheme were evaluated and modeled individually, rather than as the summarized Charlson comorbidity score, because the weighting of the original Charlson index does not accurately reflect the prognostic importance of each constituent comorbidity for CABG surgery. ${ }^{9}$ Four additional clinical variables were defined by consensus assignment of ICD-9 codes as follows: prior CABG surgery (v45.8), ventricular aneurysm (414.1), hypertension (401 through 405), and unstable angina (411.1).

The diagnosis-type indicator field in the CIHI data was then evaluated for each of the above variables, to determine the frequency with which each variable was coded as diagnosis type 2, designating complications that arose after hospital admission. In the case of CABG surgery, a type 2 diagnosis would most often represent postoperative complications (but see the caveats mentioned in Discussion).

We also studied 5 additional variables that are not affected by the diagnosis-type indicator: age, gender, urgent admission status (defined dichotomously from the "admission-type field" in CIHI data), combined CABG/valve procedure (defined by the presence of CCP codes 47.0 through $47.9^{6}$ ), and evidence of an angioplasty procedure on the same admission (defined by CCP codes 48.01 through 48.09). These additional variables were modeled with the 21 candidate variables mentioned above as potential predictors of death after CABG surgery.

There were no missing data in the resulting database, because clinical variables were defined as present (i.e., coded " 1 ") when a specific ICD-9 diagnosis code or CCP code was present, and by default were considered absent (coded "0") when the code in question was absent.

\section{Analysis}

We performed 2 separate logistic regression analyses predicting in-hospital mortality in which we either ignored (model A) or used (model B) the diagnosis-type indicator to define each of the 21 clinical variables that are based on ICD-9 diagnosis codes. In the case of model B, we only considered a variable to be present if its diagnosis type was not "2." For both models, we only considered variables that appeared in at least 10 cases (only 1 variable, human immunodeficiency virus disease, was excluded from the analysis on this basis), and we only retained those that were significantly associated with mortality $(P \leq .05)$. Model discrimination was assessed by the c statistic (area under the receiver operating characteristic curve).
The 2 resulting logistic regression models were then used to calculate 4-year risk-adjusted in-hospital mortality rates for each of the 23 Canadian hospitals performing CABG surgery in the years studied. This was done by calculating an expected mortality rate for each hospital (E) as the mean of model-predicted probabilities of death for cases in each hospital. The observed death rate $(O)$ for each hospital was then divided by its expected death rate to yield an $\mathrm{O} / \mathrm{E}$ ratio, which in turn was multiplied by the overall death rate for the 4 years studied to generate a hospitalspecific risk-adjusted mortality rate.

\section{RESULTS}

We studied a total of 50,357 CABG surgery cases performed in 23 hospitals located in 8 Canadian provinces. The mean age of patients was 63.3 years, $22.9 \%$ of cases were female, $48.0 \%$ were elective, and $10.0 \%$ were combined $\mathrm{CABG}$ and valve procedures. The overall in-hospital mortality rate for these CABG cases was 3.6\%.

Table 1 presents the extent to which individual clinical risk variables are present at baseline when coded, and therefore not coded as complication diagnoses in the diagnosis-type indicator field. Most of the variables studied (14 of 21) were present at baseline in over $97 \%$ of the instances in which they were coded. However, 7 variables were frequently coded as complications arising after admission. These included renal disease (coded as a complication $13 \%$ of the time), recent myocardial infarction (15\% of the time), peptic ulcer disease (15\%), congestive heart failure (17\%), cerebrovascular disease (26\%), hemiplegia (34\%), and severe liver disease (35\%).

Table 2 presents the multivariable odds ratios for each of the clinical variables that were significantly associated with in-hospital mortality in our multivariable analyses. In model A, the clinical variables were defined by the ICD-9 codes, whereas the clinical variables in model B were defined using the diagnosis-type indicator as those that were present at time of hospital admission (not complications). In most instances, the multivariable odds ratios drop, sometimes considerably, when the diagnosis-type indicator is taken into account to exclude complications. The magnitude of change in the odds ratios for model $\mathrm{A}$ versus model $\mathrm{B}$ is greatest for variables that were frequently coded as complication diagnoses-especially recent myocardial infarction, cerebrovascular disease, severe liver disease, and renal disease. It should be noted, however, that it is only for recent myocardial infarction and cerebrovascular disease that the odds ratios' $95 \%$ confidence intervals are nonoverlapping across models. The c statistic was considerably higher for model A (0.816) than for model B (0.759).

Table 3 presents risk-adjusted 4-year mortality rates for each of the 23 hospitals, as calculated using model A and model B. Risk-adjusted mortality rates were sometimes similar according to the two models, but for a number of hospitals, mortality rates and rankings changed, 
Table 1. Extent to which Individual Clinical Risk Variables are Present at Baseline when Coded $(N=50,357)$

\begin{tabular}{lcc}
\hline \hline Clinical Variable & Prevalence, \% & $\begin{array}{c}\text { Proportion of Instances where Present at } \\
\text { Baseline i.e., Not Coded as a Complication, \% }\end{array}$ \\
\hline Prior CABG surgery & 3.0 & 100 \\
Rheumatological disease & 0.6 & 100 \\
Mild liver disease & 0.08 & 100 \\
Metastatic cancer & 0.07 & 100 \\
Human immunodeficiency virus disease & 0.002 & 100 \\
Unstable angina & 34.4 & 99 \\
Diabetes & 16.2 & 99 \\
Chronic lung disease & 7.3 & 99 \\
Diabetes with complications & 1.1 & 99 \\
Ventricular aneurysm & 1.8 & 99 \\
Neoplasm & 0.8 & 99 \\
Hypertension & 28.8 & 98 \\
Peripheral vascular disease & 4.2 & 97 \\
Dementia & 0.06 & 97 \\
Renal disease & 1.2 & 87 \\
Recent myocardial infarction & 16.5 & 85 \\
Peptic ulcer disease & 1.0 & 85 \\
Congestive heart failure & 9.1 & 83 \\
Cerebrovascular disease & 5.1 & 74 \\
Hemiplegia & 0.6 & 66 \\
Moderate to severe liver disease & 0.06 & 65 \\
\hline
\end{tabular}

occasionally quite considerably, when diagnosis type was taken into account. Mortality rankings increased for 10 hospitals, decreased for 9 hospitals, and remained the same for only 4 hospitals. Notably, rankings for 3 of the hospitals changed by 5 positions.

\section{DISCUSSION}

This study with Canadian administrative data on CABG surgery cases identifies a number of clinical variables that frequently carry a diagnosis type 2 , indicating that these variables are often complications rather than baseline comorbidities. The inclusion of complications in analyses of risk-adjusted mortality rates is conceptually problematic, because such analyses should only adjust mortality rates for baseline severity of illness, not complications that arise from surgery. When we excluded such complication diagnoses from a risk adjustment analysis of Canadian CABG surgery mortality rates, the death rates and rankings of individual hospitals changed considerably. We suspect that the change in results relates to an "overadjustment" phenomenon, where some hospitals' death rates are adjusted to account for conditions that represent adverse events after surgery rather than baseline severity of illness. This over-adjustment would tend to not be uniform across hospitals when adverse event rates differ.

Our results are fundamentally very similar to those of Shapiro et al., ${ }^{10}$ who compared the results of risk adjustment analyses that either used "full other diagnoses" or "limited other diagnoses" to compare mortality rates in municipal and voluntary hospitals in New York City. These investigators found that the use of a "limited other diagnoses" strategy that excludes secondary diagnoses that are often complications (e.g., septicemia or pulmonary embolism) yields very different hospital mortality results in risk adjustment analyses than does a "full other diagnoses" strategy. While the approach used by Shapiro et al. ${ }^{10}$ is entirely appropriate within the constraints of administrative databases, it provides only a partial solution to the challenge of separating pre-existing conditions from complications. It is often difficult to categorize diagnoses as being either always pre-existing, or always complications. Many important diagnoses, such as congestive heart failure or myocardial infarction, can arise both as pre-existing conditions and complications, in which case a diagnosistype indicator would be necessary to make this distinction when such diagnoses are coded.

We propose that our findings are important from a few perspectives. Researchers who use administrative data that do not include a diagnosis-type indicator can be assured that the first 14 comorbidity variables presented in Table 1 are predominantly present at baseline and therefore highly appropriate as indicators of baseline severity of illness in risk adjustment analyses. On the other hand, our results also suggest that researchers will have to be careful with at least 7 variables (renal disease, recent myocardial infarction, peptic ulcer disease, congestive heart failure, cerebrovascular disease, hemiplegia, and severe liver disease), as these were coded as complications between $13 \%$ and $35 \%$ of the time in our CABG surgery database.

Researchers and general clinical readers will also notice in Table 2 that the odds ratios from a logistic regression analysis predicting mortality change when complication diagnoses are excluded from variable definitions. Notably, it is the odds ratios of variables that are often complications that tended to change most markedly 
Table 2. Odds Ratios Derived from Multivariable Analyses that Either Ignore (Model A) or Utilize (Model B) the Diagnosis-type Indicator to Exclude Complication Diagnoses

\begin{tabular}{|c|c|c|}
\hline Clinical Variable & $\begin{array}{c}\text { Model A } \\
\text { Odds Ratio } \\
\text { (95\% Confidence } \\
\text { Interval) }\end{array}$ & $\begin{array}{c}\text { Model B } \\
\text { Odds Ratio } \\
\text { (95\% Confidence } \\
\text { Interval) }\end{array}$ \\
\hline \multicolumn{3}{|l|}{ Moderate to severe } \\
\hline liver disease & $15.8(7.4$ to 34.0$)$ & 11.7 (4.5 to 30.1$)$ \\
\hline Metastatic cancer & $3.9(1.3$ to 11.4$)$ & $3.7(1.3$ to 10.5$)$ \\
\hline Renal disease & 3.7 (2.9 to 4.6$)$ & $3.2(2.5$ to 4.1$)$ \\
\hline Hemiplegia* & - & $2.5(1.6$ to 4.0$)$ \\
\hline $\begin{array}{l}\text { Recent myocardial } \\
\text { infarction }\end{array}$ & 4.2 (3.7 to 4.6$)$ & 2.3 (2.1 to 2.6 ) \\
\hline Congestive & & \\
\hline heart failure & 2.5 (2.2 to 2.8$)$ & 2.2 (1.9 to 2.5$)$ \\
\hline Prior $\mathrm{CABG}$ & & \\
\hline surgery & $1.9(1.5$ to 2.4$)$ & $1.9(1.5$ to 2.4$)$ \\
\hline $\begin{array}{l}\text { Ventricular } \\
\text { aneurysm }\end{array}$ & $1.8(1.4$ to 2.4$)$ & $1.8(1.4$ to 2.4$)$ \\
\hline Peripheral & & \\
\hline vascular disease & $1.9(1.6$ to 2.3$)$ & 1.8 (1.5 to 2.2$)$ \\
\hline $\begin{array}{l}\text { Cerebrovascular } \\
\text { disease }\end{array}$ & 2.6 (2.2 to 3.0$)$ & 1.5 (1.3 to 1.9$)$ \\
\hline $\begin{array}{l}\text { Combined CABG/ } \\
\text { valve procedure }^{\dagger}\end{array}$ & 2.7 (2.3 to 3.0$)$ & 2.6 (2.3 to 2.9 ) \\
\hline $\begin{array}{l}\text { Angioplasty on } \\
\text { same admission }^{\dagger}\end{array}$ & $1.8(1.5$ to 2.2$)$ & $2.0(1.7$ to 2.4$)$ \\
\hline Age (10-y increments $)^{\dagger}$ & $1.4(1.4$ to 1.5$)$ & $1.5(1.4$ to 1.5$)$ \\
\hline Female gender ${ }^{\dagger}$ & $1.4(1.2$ to 1.5$)$ & $1.4(1.3$ to 1.6$)$ \\
\hline $\begin{array}{l}\text { Urgent hospital } \\
\text { admission }^{\dagger}\end{array}$ & $1.3(1.1$ to 1.4$)$ & 1.5 (1.4 to 1.7$)$ \\
\hline
\end{tabular}

* Hemiplegia was not significantly associated with in-hospital mortality in model A (i.e., $\mathrm{P}>$.05).

${ }^{\dagger}$ Indicates variables whose definitions did not involve ICD-9 diagnosis codes or the diagnosis-type indicator.

when the diagnosis-type indicator was used to exclude complications from the analysis. In particular, the odds ratios for two relatively common clinical variables, recent myocardial infarction and cerebrovascular disease, differed significantly across models with nonoverlapping confidence intervals. Also of note is the higher c statistic of 0.816 for the model that includes complications versus only 0.759 for the model that excludes complications. The performance of model $\mathrm{B}$ compares very favorably to prior work done using administrative data (e.g., $c=0.75$ in earlier work with Massachusetts administrative data ${ }^{11}$ ) and also to prior modeling work done with more detailed clinical databases (e.g., $c=0.79$ for the New York State CABG model ${ }^{12}$ and $\mathrm{c}=0.76$ for the Northern New England CABG surgery model ${ }^{13}$ ). The higher $\mathrm{c}$ statistic for the model with complications (model $\mathrm{A}$ ) is a finding that indicates a greater ability to predict mortality when complications are included in the model-an expected finding, because complications are usually part of the chain of adverse clinical events that often leads to death. As mentioned earlier, the inclusion of complication diagnoses, although tempting from the standpoint of model performance, is problematic when the objective of the analysis is to only adjust mortality rates for differences in baseline severity of illness. ${ }^{14,15}$ Accordingly, we recommend general caution in the interpretation of specific odds ratios and model performance in multivariable analyses performed on administrative data if complication diagnoses are not, or cannot, be excluded.

It should be noted, however, that the type of variables included in a multivariable model depends on the planned use of the model. If the objective is simply to predict who lives and dies after surgery, it would be entirely reasonable to use a model that includes all available clinical variables to predict in-hospital death, regardless of the pre- or postoperative timing of these variables. On the other hand, if the objective of modeling is to develop a risk-adjustment model that will adjust outcome rates to control for preoperative severity of illness (i.e., the usual objective in risk-adjusted outcome analyses), then a model that only includes baseline variables is conceptually preferable.

From the perspective of care providers and consumers of health care "report cards," the results in Table 3 are important, because they demonstrate a change in adjusted death rates and rankings for several hospitals when complication diagnoses are excluded from the risk adjustment analysis. The inability to exclude complication diagnoses is a limitation that has been frequently cited by researchers evaluating report cards derived from administrative data. ${ }^{1,14,16}$

Of course, the mere inclusion of a diagnosis-type indicator does not guarantee the validity of outcome report cards derived from administrative data. A number of other concerns persist such as the selective undercoding of some secondary diagnoses in patients who die, ${ }^{17}$ and differences in administrative data quality across hospitals. Furthermore, the diagnosis-type indicator in Canadian administrative data simply indicates that a diagnosis arose some time after admission, but not necessarily after surgery. It is therefore possible that some of the excluded complication diagnoses were actually complications of preoperative medical care (e.g., arising from care in a coronary care unit) and not surgical complications. Also, it is conceivable that some of the coded complication diagnoses may actually represent chronic diagnoses that were present at the time of admission, but only diagnosed later in the hospitalization. (This latter scenario may explain our finding for the moderate to severe liver disease variable where, for example, the occurrence of postoperative hepatic encephalopathy might lead to liver disease being coded as a complication.) In both scenarios, the coded complication diagnoses would have been present preoperatively and thus fair representations of baseline severity of illness for CABG surgery. These two potential coding scenarios highlight the need for studies that formally evaluate the validity of Canadian diagnosis-type indicator coding relative to a criterion standard (medical chart review). In the absence of such validation studies, however, we would nonetheless propose that the exclusion of 
Table 3. Risk-adjusted In-hospital Mortality Rates for 23 Canadian Hospitals Calculated Using Variable Definitions that Either Ignore (Model A) or Use (Model B) the Diagnosis-type Indicator*

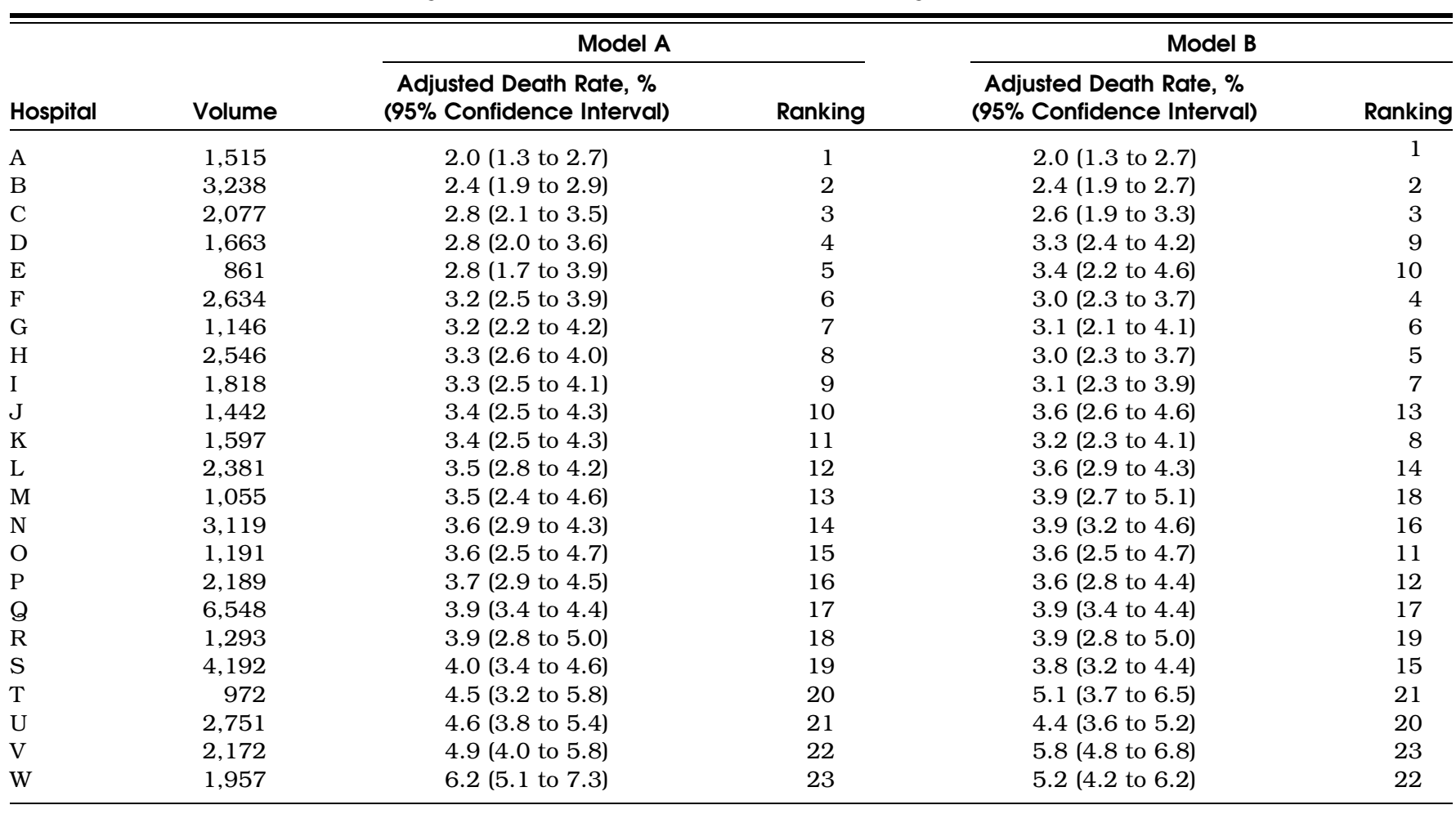

* Adjusted death rates differ significantly across the 23 hospitals $(\mathrm{P}<.001$ for both models, as determined from increments in -2 log likelihood $\chi^{2}$ values in models with versus models without dummy variables for the 23 hospitals).

complication diagnoses based on the diagnosis-type indicator improves the face validity, and ultimately perhaps also the construct validity, of risk adjustment analyses based on administrative data.

The lack of data on the accuracy of the diagnosistype indicator is an important overriding limitation to our study, and there is clearly a need for future research into the validity of indicator coding evaluated against a criterion standard. Diagnosis-type coding requires coders to make difficult clinical judgements regarding the timing and importance of clinical diagnoses. We anticipate that these judgements are often difficult to make, and in some instances perhaps even impossible. Of concern, we know that there have been anecdotal reports from New York (where diagnosis-type indicators have been introduced) that suggest possible problems with the accuracy of the timing indicators being coded in that state. Reassuringly, however, we see in our data (Table 1) that Canadian diagnosis-type coding at least has face validity, with chronic diagnoses such as diabetes, hypertension, cancer, and rheumatological disease almost always coded as baseline comorbidities, and potential complications such as myocardial infarction, peptic ulcer disease, stroke, and congestive heart failure often coded as complications.

From the perspective of health policy makers and managers of administrative data in other countries, these results point to a need for consideration of the widespread introduction of diagnosis-type indicators. In a recent review of the strengths, limitations, and future of administrative data, Iezzoni ${ }^{1}$ concluded that administrative data offer substantial and practical advantages for widespread quality assessment, but that questions about data quality currently compromise their utility, at least somewhat. Indeed, it is these quality concerns that prompted the development of impressive clinical data registries in the United States such as the prospective CABG surgery databases in New York State and Northern New England. ${ }^{12,13}$ The widespread development of such data collection initiatives is certainly one solution to avoiding the limitations of administrative data. However, the wide geographic coverage and relatively low cost of administrative data are such that they will undoubtedly continue to be used to study quality in clinical and geographic areas that are not covered by more detailed state-of-the-art clinical databases. In anticipation of the ongoing future use of administrative data to study quality of care, Iezzoni has thus proposed that clinicians and researchers will need to work together for the future to develop improved administrative databases that are "more clinically meaningful and useful." 1 The widespread introduction of a diagnosistype indicator in the United States and elsewhere may be one step toward improvement. 
This project and WAG were supported by a Population Health Investigator Grant from the Alberta Heritage Foundation for Medical Research in Edmonton, Alberta. WAG is also supported by a Government of Canada Research Chair.

\section{REFERENCES}

1. Iezzoni LI. Assessing quality using administrative data. Ann Intern Med. 1997;127:666-74.

2. Canadian Institute for Health Information (formerly HMRI). Comparative Analysis of the HMRI Database. Toronto: Canadian Institute for Health Information; 1992.

3. International Classification of Diseases, 9th revision (clinical modification). Washington, DC: Public Health Service, US Department of Health and Human Services; 1988.

4. Ghali WA, Quan H, Brant R. Coronary artery bypass grafting in Canada: national and provincial mortality trends, 1992-1995. Can Med Assoc J. 1998;159:25-31.

5. Ghali WA, Quan H, Brant R. Coronary artery bypass grafting in Canada: hospital mortality rates, 1992-1995. Can Med Assoc J. 1998;159:926-30.

6. Canadian Classification of Diagnostic, Therapeutic, and Surgical Procedures. Ottawa: Statistics Canada; 1986. Catalog number 82-562E.

7. Deyo RA, Cherkin DC, Ciol MA. Adapting a clinical comorbidity index for use with ICD-9-CM administrative databases. J Clin Epidemiol. 1992;45:613-9.

8. Charlson ME, Pompei P, Ales KL, MacKenzie CR. A new method of classifying prognostic comorbidity in longitudinal studies: development and validation. J Chronic Dis. 1987;40:373-83.
9. Ghali WA, Hall RE, Rosen AK, Ash AS, Moskowitz MA. Searching for an improved comorbidity index for use with ICD-9-CM administrative data. J Clin Epidemiol. 1996;49: 273-8.

10. Shapiro MF, Park RE, Keesey J, Brook RH. The effect of alternative case-mix adjustments on mortality differences between municipal and voluntary hospitals in New York City. Health Serv Res. 1994;29:95-112.

11. Ghali WA, Ash AS, Hall RE, Moskowitz MA. Statewide quality improvement initiatives and mortality after cardiac surgery. JAMA. 1997;277:379-82.

12. Hannan EL, Kilburn H Jr, O'Donnell JF, Lukacik G, Shields EP. Adult open heart surgery in New York State: an analysis of risk factors and hospital mortality rates. JAMA. 1990;264: 2768-74.

13. O'Connor GT, Plume SK, Olmstead EM, et al, for the Northern New England Cardiovascular Disease Study Group. A regional prospective study of in-hospital mortality associated with coronary artery bypass grafting. JAMA. 1991;266:803-9.

14. Hannan EL, Racz MJ, Jollis JG, Peterson ED. Using Medicare claims data to assess provider quality for CABG surgery: does it work well enough? Health Serv Res. 1997;31: 659-78.

15. Iezzoni LI, Ash AS, Shwartz M, Landon BE, Mackiernan YD. Predicting in-hospital deaths from coronary artery bypass graft surgery: do different severity measures give different predictions? Med Care. 1998;36:28-39.

16. Roos LL, Stranc L, James RC, Li J. Complications, comorbidities, and mortality: improving classification and prediction. Health Serv Res. 1997;32:229-38.

17. Jencks SF, Williams DK, Kay TL. Assessing hospital-associated deaths from discharge data. The role of length of stay and comorbidities. JAMA. 1988;260:2240-6.

\section{JGIM Website - Visit us online today!}

Please visit the JGIM World-Wide Website:

http://www.blackwellscience.com/journals 